An ASABE Meeting Presentation

\title{
Development of a Uniformity Controlled Granular Fertilizer Spreader
}

Tony E. Grift PhD, Assistant professor, University of Illinois. 1304 W. Pennsylvania Ave. Urbana, IL 61801, grift@uiuc.edu

Giyoung Kweon, Graduate Assistant, University of Illinois. 1304 W. Pennsylvania Ave. Urbana, IL 61801, gkweon@uiuc.edu

\author{
Written for presentation at the \\ 2006 ASABE Annual International Meeting \\ Sponsored by ASABE \\ Portland Convention Center \\ Portland, Oregon \\ 9 - 12 July 2006
}

\begin{abstract}
A method was proposed which employs control of the drop location of fertilizer particles on a spinner disc to optimize the spread pattern uniformity. The system contained an optical sensor as a feedback mechanism, which measured discharge velocity and location, as well as particle diameters to predict a spread pattern of a single disc. This pattern was combined with a mirrored pattern from a second disc to produce a complete transverse pattern of a dual disc spreader. Subsequently, the transverse pattern was used in simulations in which the patterns from both spinner disc were rotated about the disc center, which in practice is achieved by rotating segment type gates such as in the Vicon Rotaflow spreader. The transverse patterns were overlapped in software and the resulting overlapped patterns as a function of the gate angles were evaluated using three criteria, which filter offset patterns, non-robust patterns and patterns with a high sensitivity in CV around a chosen swath width. This led to maps where each gate setting was related to a quality of the spread pattern for a single mass flow value. The optimally uniform pattern was found at the minimum of the quality map. When the mass flow rate changes, the whole process needs to be repeated and a new optimal gate setting found to produce the optimal pattern at the new mass flow rate. The power of the feed gate adaptation method was shown in simulations as well as using real data from a Sulky spinner disc spreader.
\end{abstract}

The results showed that it was always possible to find a spread pattern with an acceptable CV lower than $15 \%$, even though the spread pattern was obtained from a rudimentary flat disc with straight radial vanes.

Keywords. Variable Rate Application, Precision agriculture, Optimal pattern, CV

The authors are solely responsible for the content of this technical presentation. The technical presentation does not necessarily reflect the official position of the American Society of Agricultural and Biological Engineers (ASABE), and its printing and distribution does not constitute an endorsement of views which may be expressed. Technical presentations are not subject to the formal peer review process by ASABE editorial committees; therefore, they are not to be presented as refereed publications. Citation of this work should state that it is from an ASABE meeting paper. EXAMPLE: Author's Last Name, Initials. 2006. Title of Presentation. ASABE Paper No. 06xxxx. St. Joseph, Mich.: ASABE. For information about securing permission to reprint or reproduce a technical presentation, please contact ASABE at rutter@asabe.org or 269-429-0300 (2950 Niles Road, St. Joseph, MI 49085-9659 USA). 


\section{Introduction}

The application of dry granular materials such as fertilizers, herbicides, and pesticides has been traditionally performed with equipment that was intended for constant application rates. The quality indicators of granular applicators are 1) the application rate, in kg per hectare and 2) the uniformity, expressed as a percentage. The desired application rate is chosen by the farm manager and depends on the crop, its growth stage, soil and environmental conditions. The uniformity is usually expressed in a statistical coefficient of variation (CV), the standard deviation of the (simulated) overlapped spread pattern divided by the mean. The spread pattern uniformity (CV) is ideally close to zero (which would imply perfect uniformity) but in practice, it can deviate significantly from zero. Reasons are inferior spreader designs, such as the single disc spreader, poorly adjusted spreaders, unfavorable weather conditions, and human error. Uneven application can lead to over dosage, causing material wastage, burnt crops, and potential environmental hazards, which can be seen in the field in the form of 'streaks' (Helms et al., 1987). Under application can lead to nitrogen deficient plants and limited growth.

In ground-based application predominantly spinner disc spreaders are used. Spinner type spreaders are attractive because they can cover large areas effectively; they are simple in design, reliable, inexpensive, robust, and require little maintenance. They can produce a good pattern for constant application rates if properly overlapped and calibrated. An alternative, developed in recent years, is pneumatic application where the granular material is transported through air tubes and delivered to dividers. A comparison in performance of spinner type spreaders and pneumatic spreaders is given in Fulton et al. (2004).

Fulton et al., (2001) performed a large-scale investigation on the rate and uniformity of a spinner type spreader using the ASAE 341.2 standardized collection tray method. The spreader was started at a low application rate of $56 \mathrm{~kg} / \mathrm{ha}$, which was gradually increased to the maximum rate of $168 \mathrm{~kg} / \mathrm{ha}$. The resulting spread pattern changed from a desirable Gaussian (bell shape) at the low application rate to an M-shape at the medium setting to a very undesirable $\mathrm{W}$ shape at the highest application rates. M-shapes and W-shapes make producing a uniform overlapped pattern virtually impossible and have a devastating effect on the pattern robustness (Grift, 2000). Other effects on spread pattern uniformity of a spinner type spreader are spinner height and PTO speed (Parish 2002a), as well as spreader fill level (Parish, 1999a). Parish, (2002b) confirmed the flow rate effect on spread pattern uniformity.

The studies by Fulton and Parish have shown that the spinner type spreader is not directly suited for Variable Rate Application of fertilizer unless the drop location of the particles can be adjusted in real time. Such adjustments are present in some spreaders, such as in the Vicon Rotaflow $^{1}$ design. Figure 1 shows one disc of the dual disc Rotaflow spreader that contains controls for application rate as well as uniformity by varying the drop location of the material through rotating the gates about the centers of the discs. In the Rotaflow design, the gate adjustments are made manually during calibration tests. This research aims to replace this manual adjustment by a feedback controlled system that automatically finds the correct gate angle setting for any chosen application rate at an optimal uniformity.

${ }^{1}$ Mentioning of brand names does not imply endorsement of products either by the authors or the University of Illinois. 


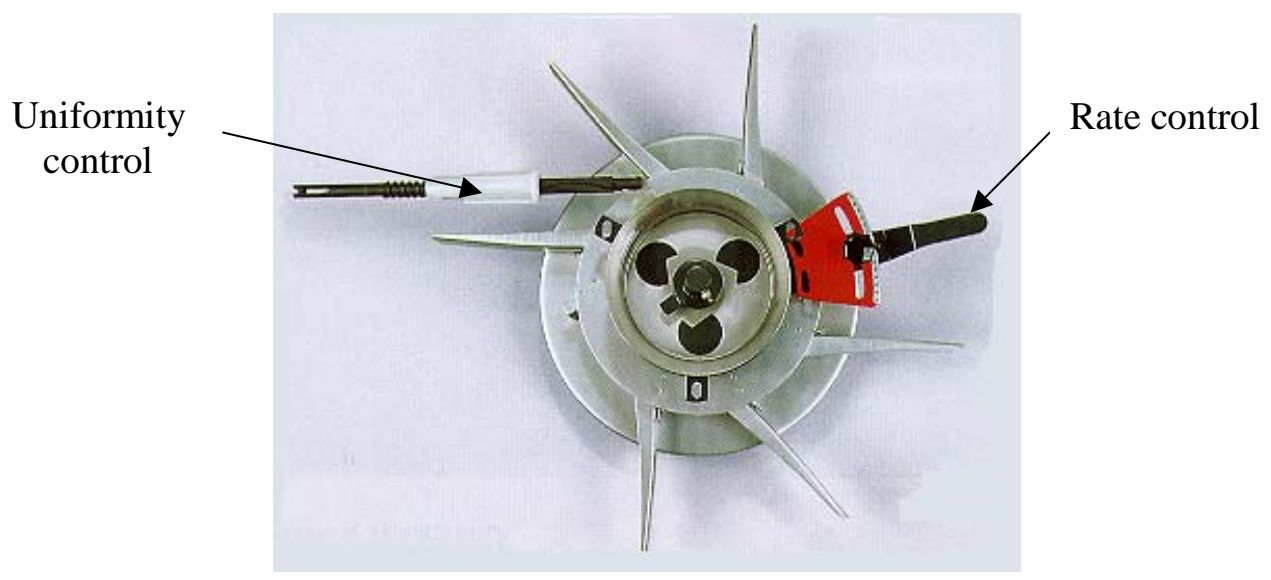

Figure 1. Vicon Rotaflow design with rate and uniformity controls.

The objectives of this study were:

1. To investigate the effect of mass flow rate changes on pattern uniformity through simulation using models from the literature

2. To develop a feed gate adaptation algorithm that optimizes the spread pattern uniformity by varying the drop locations of particles on the discs

3. To evaluate the performance of the feed gate adaptation algorithm using a fertilizer spreader test bed

4. To develop a generic platform which allows studying fundamental particle behavior on the disc as well as developing alternative feed gate control algorithms 


\section{Materials and Methods}

The first step in studying the effect of fertilizer mass flow on the spread pattern was to simulate the particle trajectories after being dropped from a segmented gate, which opens tangentially with respect to the disc center. For simplicity, identical spherical particles were assumed accelerated along a straight radial vane on a flat disc to comply with the model from Inns and Reece, (1962). Figure 2 shows the simulated particle trajectories for a gate opening of $15 \mathrm{deg}(\mathrm{a})$ and for an opening of $50 \mathrm{deg}$.
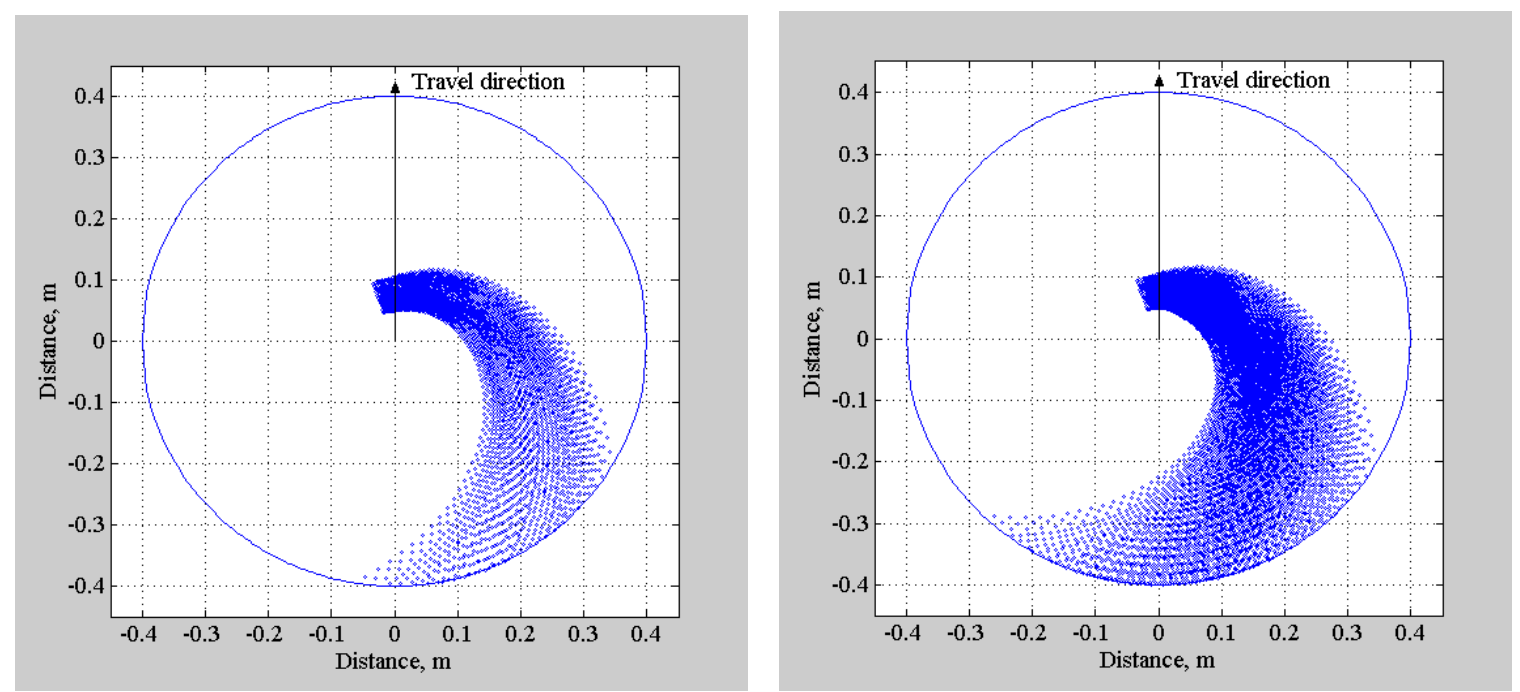

Figure 2. Identical particle flows on a disc with gate opening of $15 \mathrm{deg}$ (left) and $\mathbf{5 0 ~ d e g ~ ( r i g h t ) . ~}$

It is clear from the simulation that the varying mass flow has a pronounced effect on the discharge location of the particles and consequently, on the resulting spread pattern.

The second step was simulating the trajectories of particles in air. Figure 3 shows the simulated spread pattern of a dual disc spreader where the pattern from a single disc (figure 2) was combined with a mirror image pattern from the second disc. The landing locations were computed using a ballistic model (Mennel and Reece, 1963), which accepted the simulated discharge velocity and location as well as the particle diameter as inputs. 


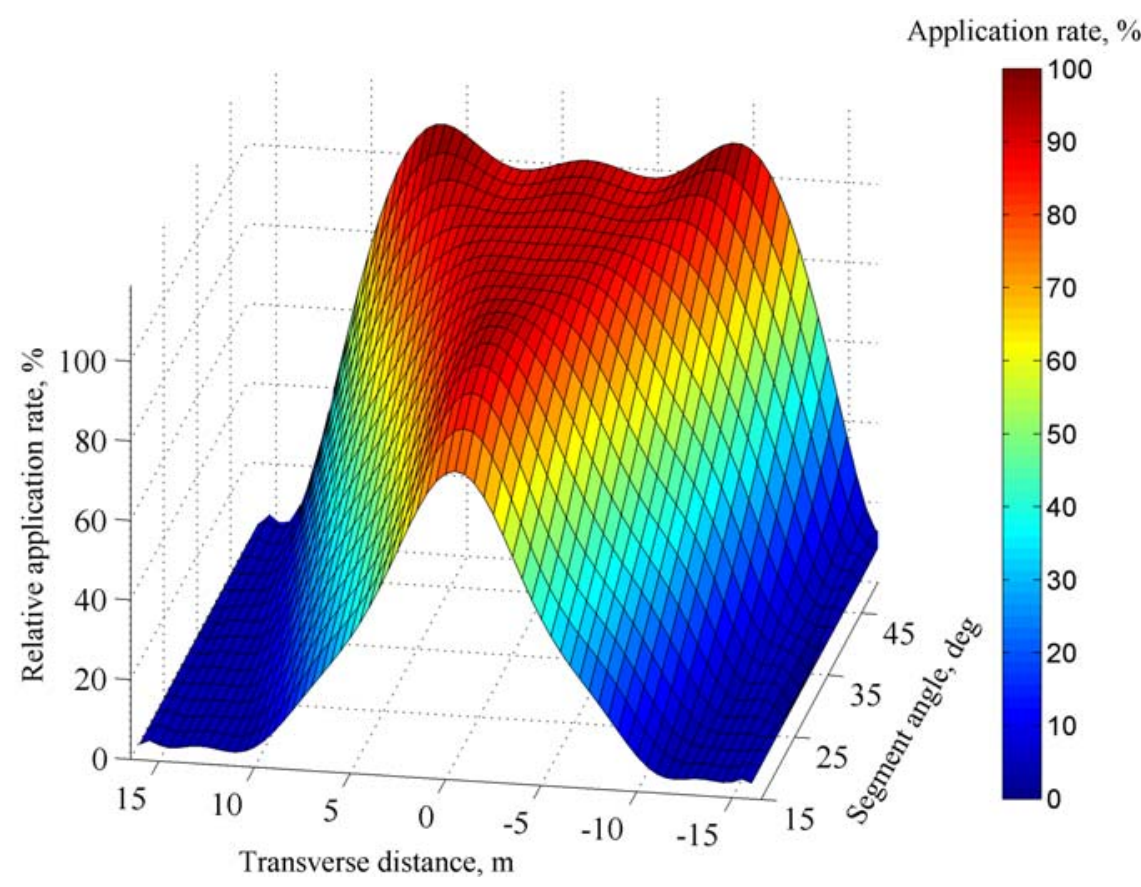

Figure 3. Simulated transverse spread pattern from dual discs showing an ideal Gaussian at a low flow rate (15 deg segment angle) and an M-shape at a high flow rate (50 deg segment angle).

Grift (2000) claimed that the most desirable spread pattern is Gaussian shaped and indeed the pattern at a low flow rate is similar in figure 3. However for higher flow rates, the pattern becomes severely distorted to an undesirable M-shape at the maximum gate angle (maximum flow rate). This shape makes producing a uniform spread pattern without any form of feed gate adaptation a daunting task.

\subsection{Feed gate adaptation development}

The feed gate adaptation algorithm was based on simulating the overlapped dual disc pattern for a large number of gate settings and judging the quality of the pattern by three criteria. Firstly a pattern must be centered in the driveline. For this criterion the weighted arithmetic mean value of the single transverse (not overlapped) pattern was computed, which yields a low value for a centered pattern. Secondly, the overlapped pattern's robustness was computed (Grift, 2000) which is a global criterion related to the 'forgivingness' of a pattern; a pattern with a low robustness allows for errors in the swath width whilst maintaining an acceptable uniformity. Thirdly, an additional local criterion was used which consisted of the average CV of the overlapped pattern in an arbitrary 90-110\% interval around a chosen swath width.

Since the three criteria yield low values for good patterns, the optimal pattern can be found at the minimum of the sum of the three criteria. Figure 4 shows the sum of the three criteria termed a 'quality map' and indeed the lowest values can be found where the gate settings are equal, implying that symmetric patterns are of high quality. Note that the graph only represents a single application rate. When the application rate varies, the graph must be regenerated and new optimal gate settings found. 


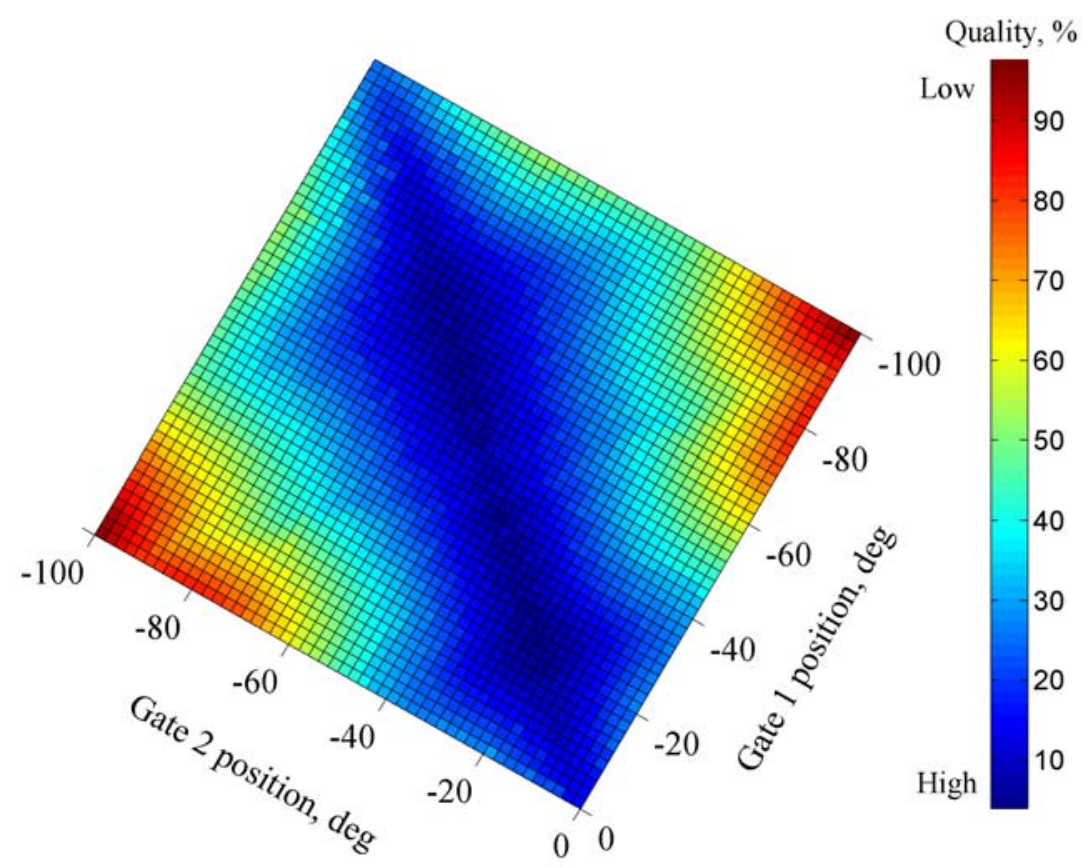

Figure 4. Sum of three criteria with the varying gate positions in dual disc case.

The feed gate adaptation method has shown that the M-shape effect can be avoided by finding the optimal gate settings according to the three criteria. Figure 5 shows the same initial Gaussian pattern for low flow rates as in figure 3, but after applying the feed gate adaptation method, the pattern remains close to a Gaussian and the spread width is virtually constant for higher application rates. This simulation demonstrates that changing the drop location of the particles by rotating the feed gate about the disc center is indeed capable of producing high quality spread patterns, independent of the mass flow rate.

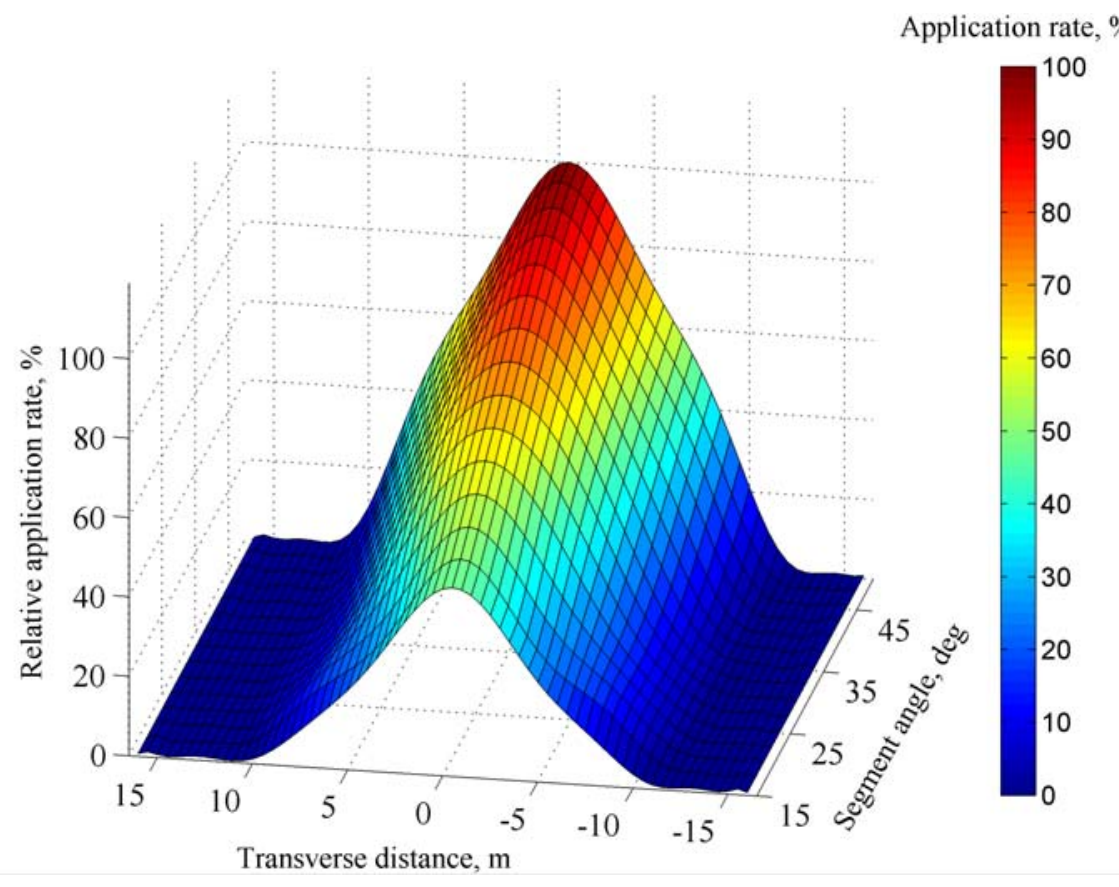

Figure 5. Simulated single transverse spread pattern after application of the feed gate adaptation algorithm. 
In summary, the following steps are used in the feed gate adaptation method for a single mass flow rate:

1. Determine the discharge velocity, angle and particle diameters from a single disc and predict a two-dimensional particle landing location pattern

2. Rotate the single disc pattern to a chosen initial gate angle about the disc center

3. Combine the single disc pattern with a mirror pattern to produce a transverse spread pattern of a dual disc spreader

4. Overlap the transverse spread pattern at a chosen swath width to obtain an overlapped transverse pattern in a chosen mode (Back\&Forth or RaceTrack)

5. Repeat steps 2 through 4 for all possible gate angles

6. Produce a quality map by filtering all patterns using three criteria

7. Find the optimal pattern and gate settings at the minimum of the quality map

\subsection{Feed gate adaptation implementation}

In the feed gate algorithm development, the inputs for the ballistic model, which predicted the landing locations, originated from simulating particle trajectories on a disc, resulting in discharge velocities, locations and particle diameters. In the implementation, these values are obtained from a sensor, developed by (Grift and Hofstee, 1997) who used it to predict the spread pattern of a single disc fertilizer spreader (Grift and Hofstee, 2002). To evaluate the feed gate adaptation algorithm, a half section of a Sulky spreader was used, fitted with a flat disc with straight radial vanes (figure 6).

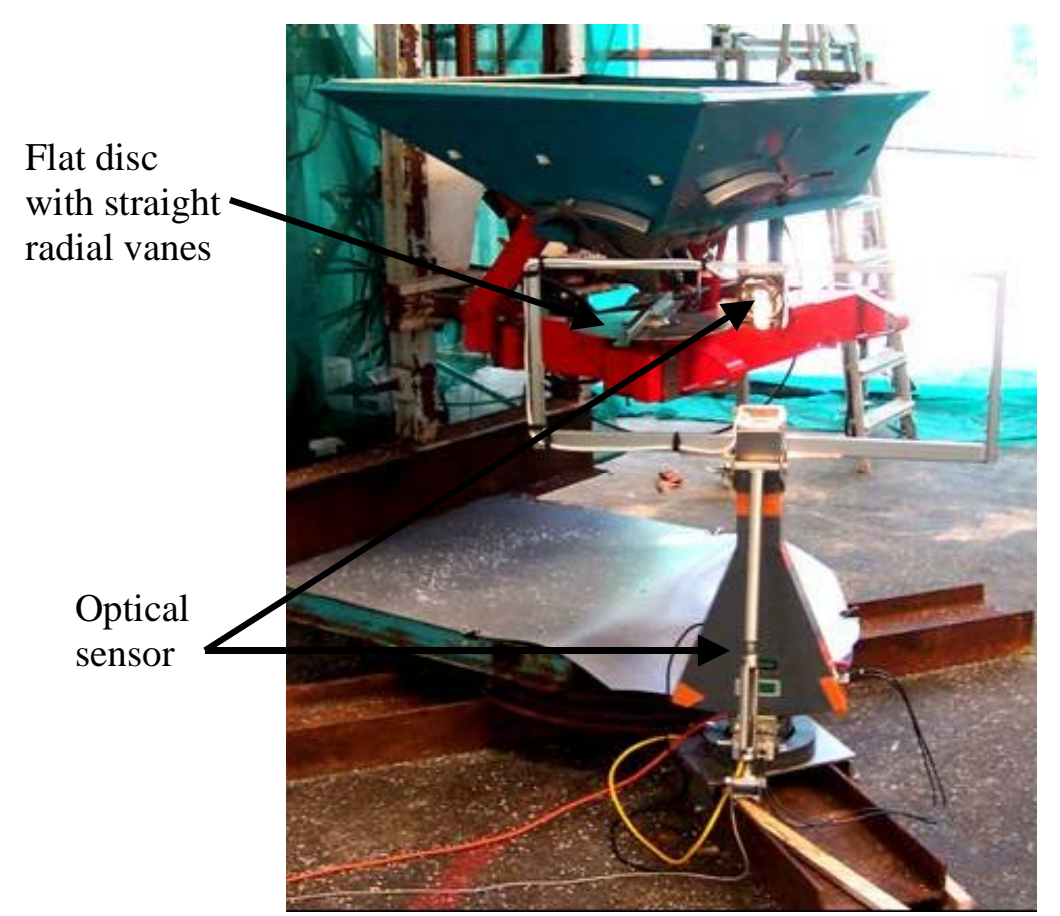

Figure 6. Test bed for feed gate adaptation algorithm validation. 


\subsection{Development of generic experimental arrangement}

A generic experimental arrangement was developed at Cemagref, Montoldre, France (figure 7). This arrangement allows for experimentation with varying spinner speeds, varying particle drop locations, different gate types and materials.

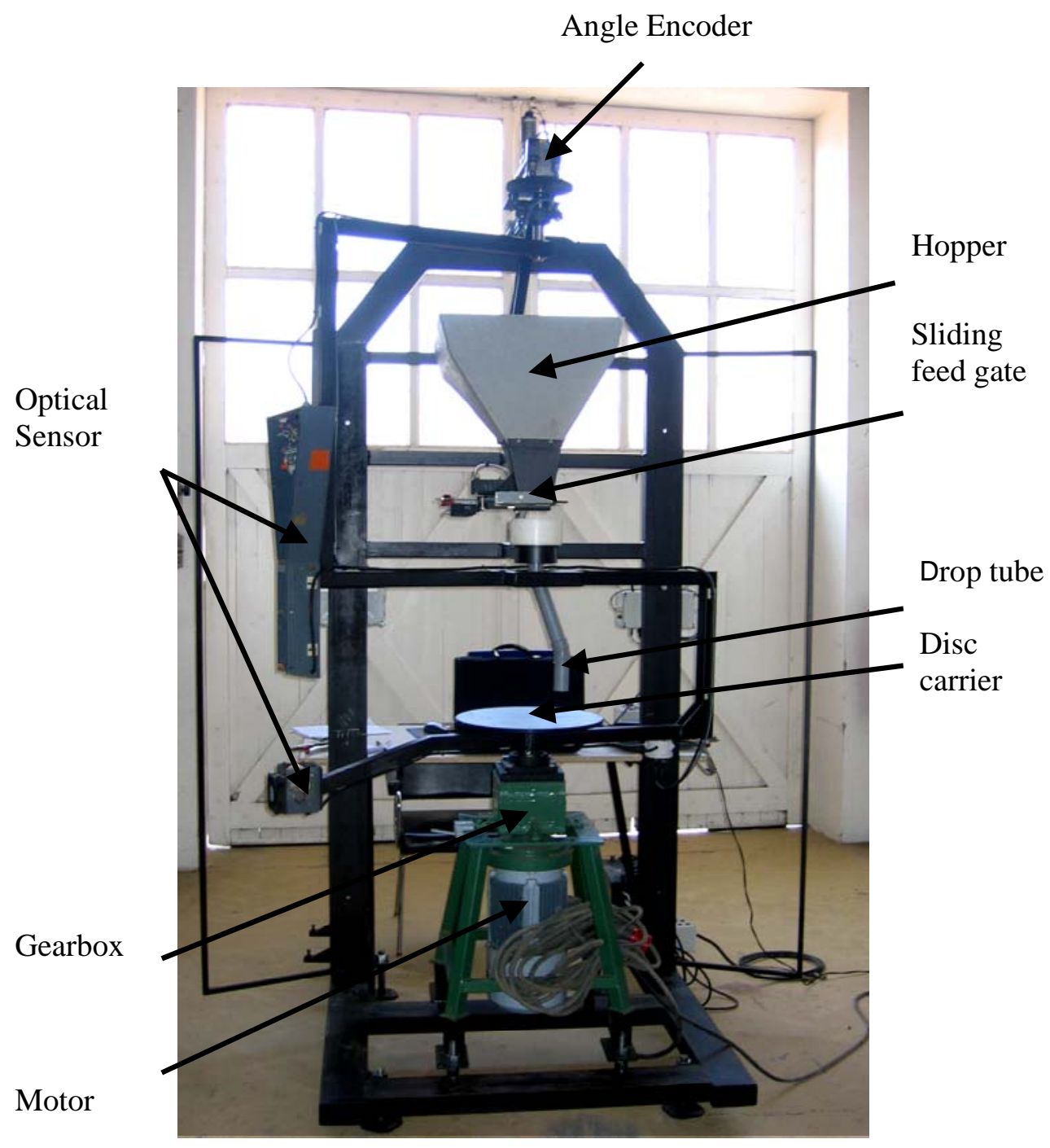

Figure 7. Photo of the generic experimental arrangement.

The optical sensor used to obtain spread patterns is an integral part of the design. To prevent particles from hitting the sensor, the light source was detached from the sensing element, such that the particles had an unobstructed path. The disc carrier can be fitted with an arbitrary disc, conical or flat with radial or pitched, curved or straight vanes. To control the speed of the disc, an electric motor (LS100, Leroy-Somer, France) and a motor speed controller (Altivas 18, Telemecanic, France) were used, which allow rotational velocities up to 2500 RPM. To rotate the sensor a DC motor (P16, Crouzet Co., France) and gears with a reduction ratio of 1:40 were used. The rotational speed of the sensor was set to 0.5 RPM. A 12-bit absolute encoder (AG 612 WKRP 4096 GRAY, Max Stegmann GmbH, Germany) was attached to the top of the axis of the rotating sensor to provide the discharge angle of the particles passing the sensor. 
To reduce the influence of the flow rate change on the spread pattern, a PVC tube was used with a sliding edge gate with a size of $40 \mathrm{~mm} \times 80 \mathrm{~mm}$. The flow rate can be controlled by changing the position of the feed gate with a linear actuator, which has a potentiometer as feedback sensor (Electrak 1, Warner linear, USA). The PVC tube can be rotated by a RC servomotor (S3305, Futuba Co., USA), which controls the drop location. The generic experimental arrangement contains the following components:

A feedback sensor which predicts the spread pattern behind the spreader

1. Actuators that control the gate opening (flow rate) as well as the particle drop location on the disc

2. An algorithm that finds the gate setting which optimizes the spread uniformity of the spreader

3. A communication network which interfaces the sensors, actuators and control hardware

Figure 8 shows the control system of the experimental arrangement.

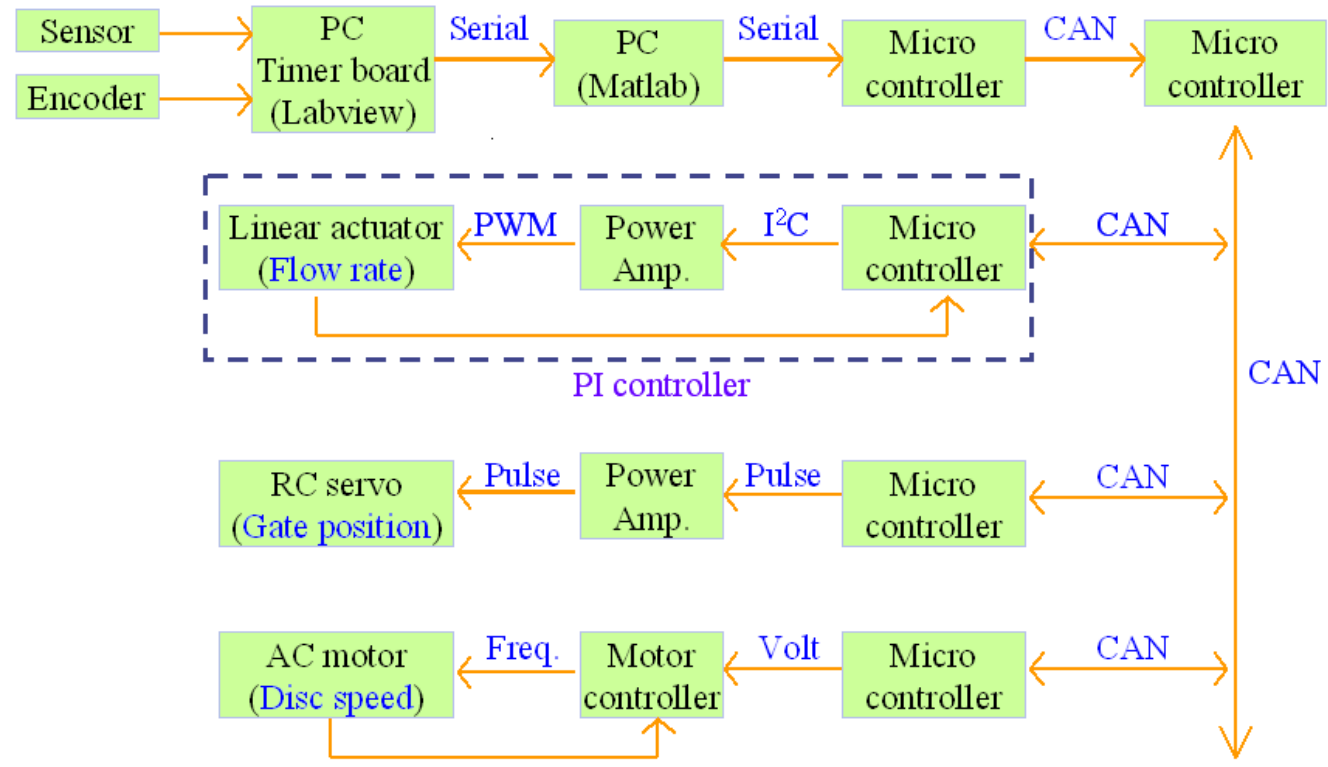

Figure 8. Control loop of the generic experiment arrangement.

The particle velocity, diameter and discharge angle information were obtained from the optical sensor and encoder using a Timer board (PCI 6602, National Instruments Co.) under control of LabVIEW (National Instruments Co.) Based on these data, a MatLab ${ }^{\circledR}$ program simulated pattern overlaps for a given swath width and subsequently generated signals to control the fertilizer drop location. Finally, actuators moved the position of the drop tube according to the set point. Several micro-controllers were used to bridge communication as well as generating pulses and output voltages to control actuators. PIC18F458 (Microchip Inc.) with Controller Area Network (Etschberger, 2001) functionality were chosen, since these allow future integration of the system with existing CAN buses on off-road equipment. Figure 9 shows a photo of the control system with three micro controllers, a laptop computer as well as two actuators. 


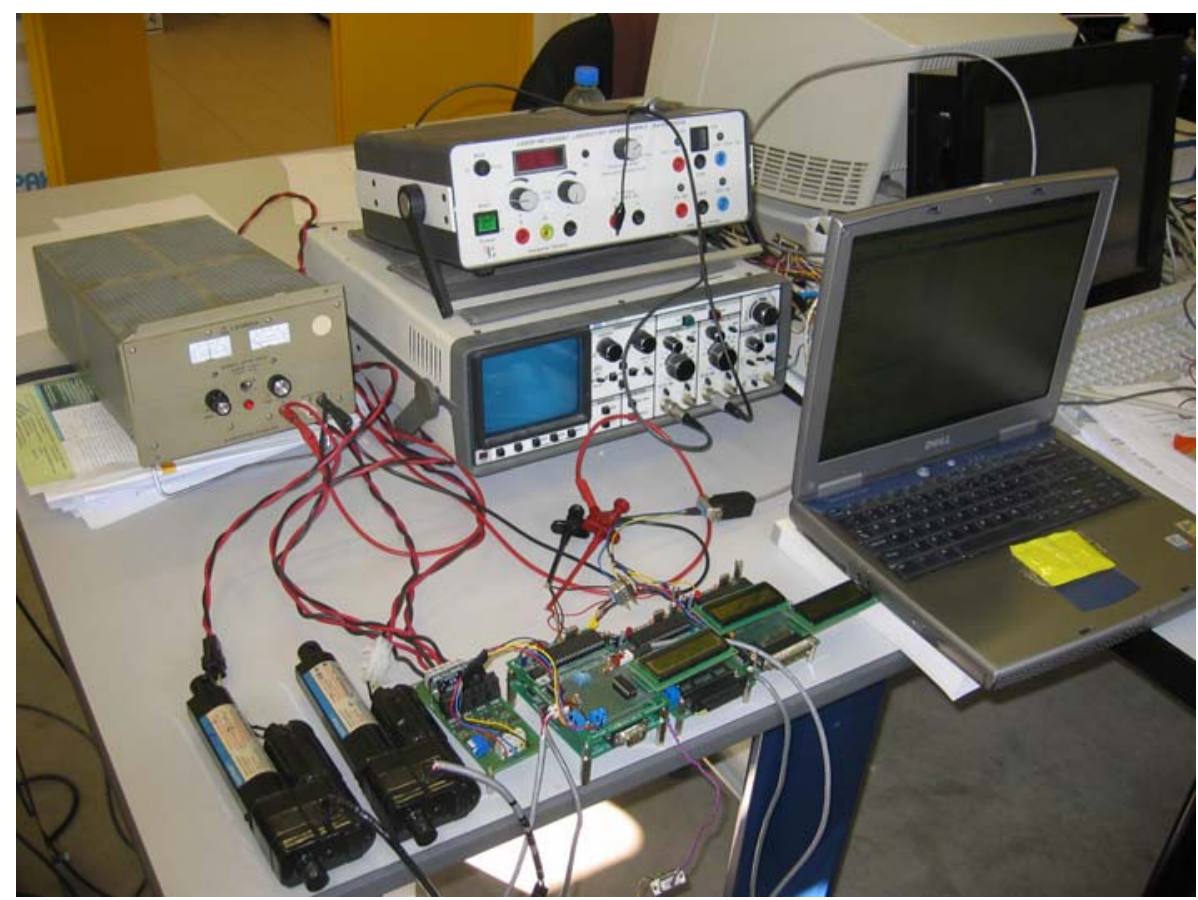

Figure 9. Photo of the linear actuators controlled by the microcontrollers through a CAN bus.

\section{Results}

Figure 10 shows the predicted spread pattern using the Sulky half section, for a mass flow of $10 \mathrm{~kg} / \mathrm{min}$, using Ammonium Nitrate fertilizer.

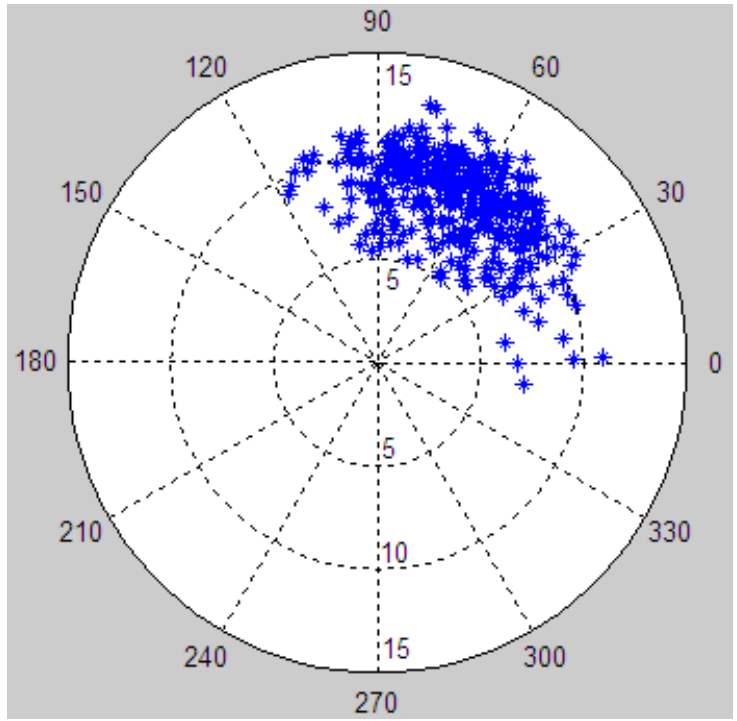

Figure10. Spread pattern produced by Sulky half section at a mass flow rate of $10 \mathrm{~kg} / \mathrm{min}$ using Ammonium Nitrate fertilizer.

From figure 10, it is clear that the pattern of a single disc is severely skewed, but this does not imply that the transverse pattern of the dual disc spreader is poor. The transverse pattern of the dual disc spreader was generated by adding a second mirrored pattern to the one shown in figure 10 at a distance equal to the design of the Sulky spreader. Figure 11 shows the quality 
map. The minima are found where the gate positions are equal (symmetric pattern) and the minimum is found at 14,14 deg for gate 1 and 2 respectively.

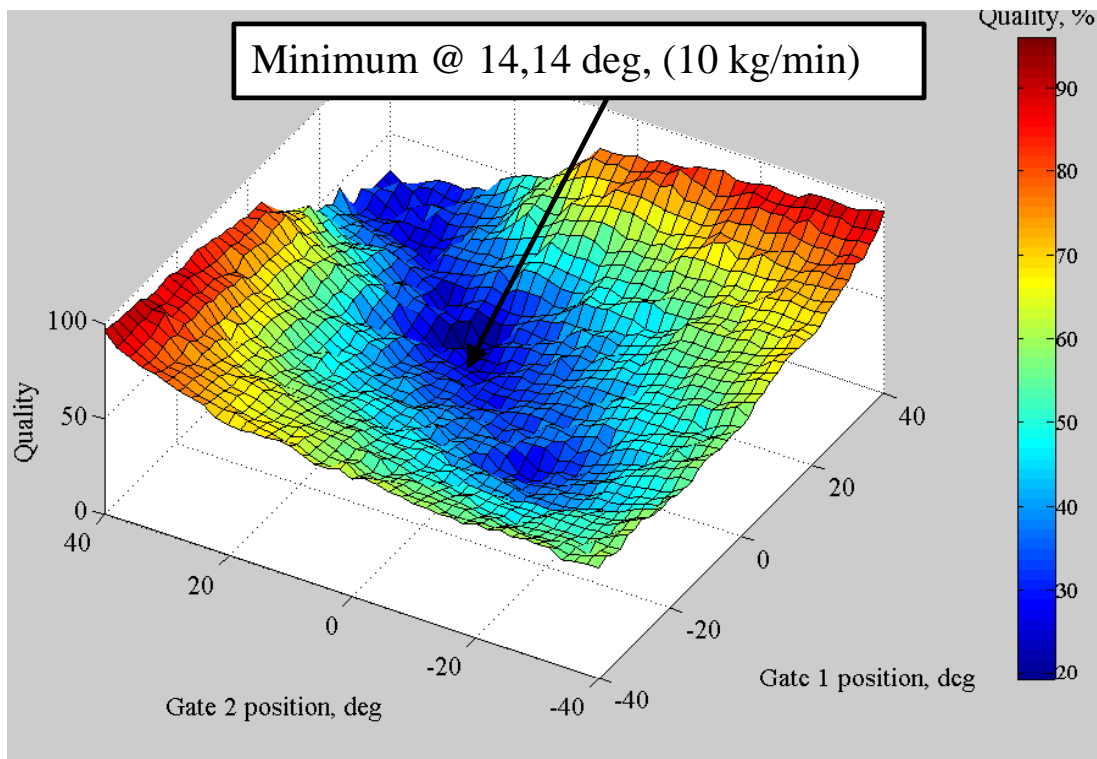

Figure 11. Quality map indicating a minimum value at gate $1,2=14 \mathrm{deg}$.

The CV- Swath width plot of the overlapped pattern of the dual disc spreader as shown in figure 12 indicates that the CV at the chosen swath width of $10 \mathrm{~m}$ and a mass flow of $10 \mathrm{~kg} / \mathrm{min}$, is $17 \%$, slightly higher than $15 \%$ (horizontal straight line) which is considered acceptable. The overlaps were carried out in Back \& Forth mode since it represents the worst-case scenario and is most popular (Parish, 1999b).

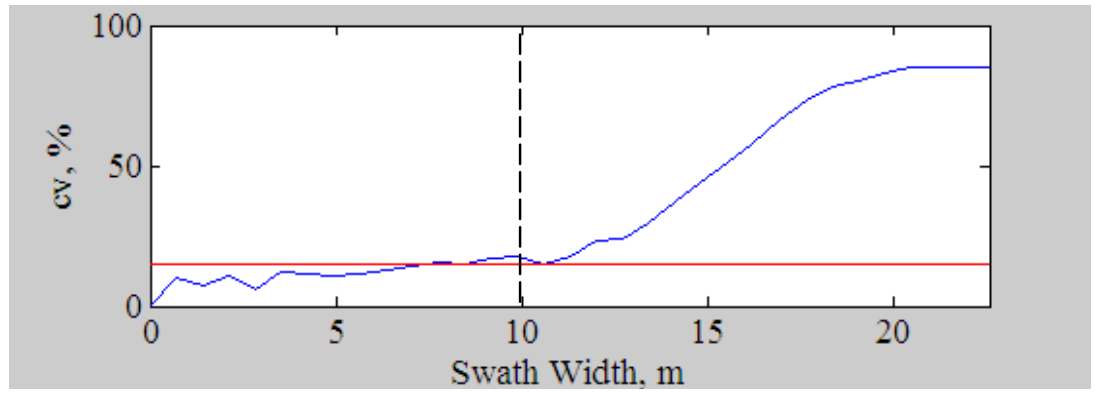

Figure 12. CV versus Swath width plot showing a CV of $18 \%$ at a swath width of $10 \mathrm{~m}$.

The spreading run, which resulted in the data shown in figure 10, was repeated for a mass flow of $15 \mathrm{~kg} / \mathrm{min}$, while keeping the gate angles constant at $14 \mathrm{deg}$. This resulted in a spread pattern, which was unacceptable, since it exhibited a CV of $27 \%$ at a swath width of $10 \mathrm{~m}$ as shown in figure 13. This result confirms that the spread pattern quality is highly dependent on the mass flow and that an adaptation algorithm is needed to control the uniformity. 


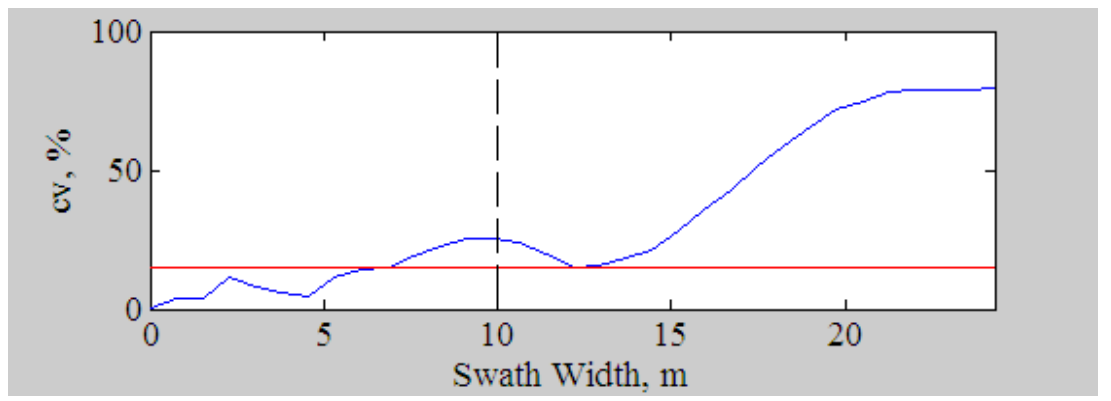

Figure 13. CV versus Swath width plot showing a CV of $27 \%$ at a swath width of $10 \mathrm{~m}$.

The feed gate algorithm was applied to the new mass flow rate of $15 \mathrm{~kg} / \mathrm{min}$ and the optimal pattern was found at a gate setting of $8 \mathrm{deg}$ for both gates as shown in figure 14 .

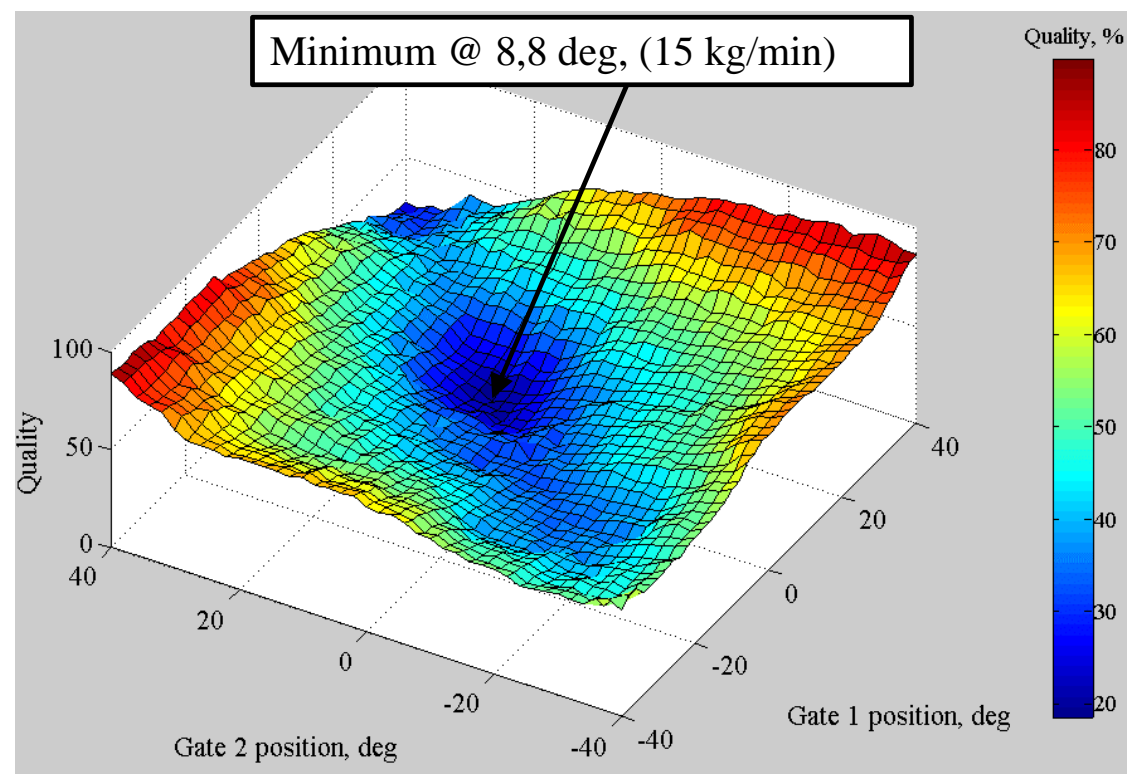

Figure 14. Quality map indicating a minimum value at gate 1,2 = 8 deg.

The resulting spread pattern uniformity as a function of swath width is shown in figure 15 . It is clear that the uniformity has greatly improved compared to the original 14 deg gate setting version (figure 13), not only locally at $10 \mathrm{~m}$ where the $\mathrm{CV}$ is $15 \%$, but the pattern has a lower CV for a range of swath widths, which makes it more robust.

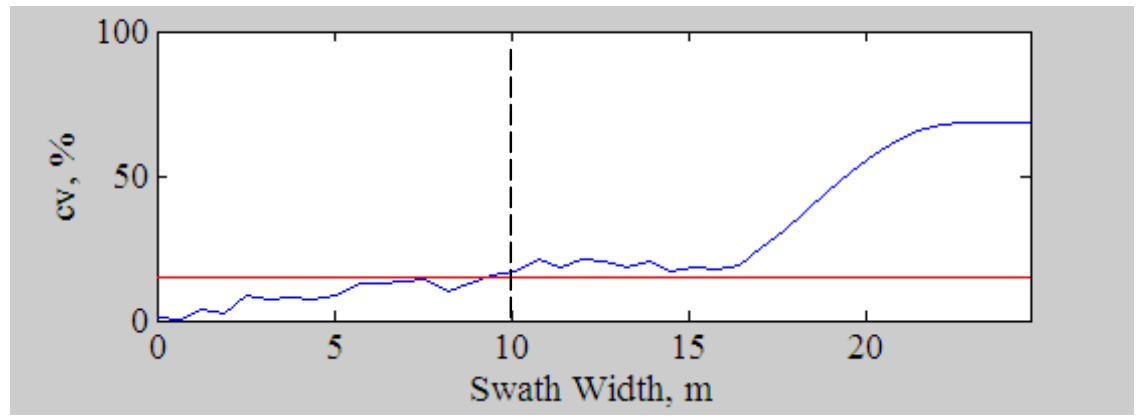

Figure 15. CV versus Swath width plot showing a CV of $15 \%$ at a swath width of $10 \mathrm{~m}$. 


\section{Conclusions and discussion}

This paper describes the development of a uniformity controlled spinning disc granular fertilizer spreader. Simulations showed that it is possible to obtain highly uniform patterns by rotating the drop location of the fertilizer particles about the center of the disc.

The procedure is that for every gate setting, an optical sensor predicts the spread pattern on the ground and by rotating this pattern and simulating overlaps, a CV-swath width curve of overlapped patterns can be computed. These then serve as inputs for a three-criteria filter, which gives low values for high quality patterns.

The feed gate adaptation algorithm was tested on a Sulky half section spreader with a single flat disc with straight radial vanes. First for a setting of $10 \mathrm{~kg} / \mathrm{min}$, the spread pattern was determined using an optical sensor. This pattern was rotated to an initial value of the gate, and then added to a mirror pattern to obtain a dual disc spread pattern, and this pattern was overlapped in software to obtain an overlapped transverse pattern. This procedure was repeated for all possible gate positions. After filtering all patterns with three quality criteria, a reasonable pattern was found at gate angles of 14 deg resulting in a pattern with a CV of $17 \%$ at a swath width of $10 \mathrm{~m}$. The same gate settings were used for a mass flow of $15 \mathrm{~kg} / \mathrm{min}$ and as expected, the uniformity deteriorated to a CV of $27 \%$ for the same $10 \mathrm{~m}$ swath width. This confirmed the findings from simulations: the pattern quality is highly dependent on the application rate. For the $15 \mathrm{~kg} / \mathrm{min}$ pattern, the feed gate adaptation algorithm was applied once more and the result was that the new gate angles needed to be set to 8 deg for optimal uniformity. These gate settings produced a spread pattern with a CV of $15 \%$, and in fact the overall spread pattern quality (robustness) was improved.

The current approach was limited to both spinning discs rotating at identical rotational velocities. Therefore, it was valid to assume that the patterns from both discs were mirror images and the quality plots became symmetric. However, it is conceivable that in the future asymmetric patterns are required, particularly for border spreading, to avoid waterways and other environmentally sensitive areas. The approach as presented here will work equally well, however both discs will require feedback sensors and the resulting quality plots will be asymmetric.

This research showed a procedure that was based on repeating the feed gate adaptation algorithm for every newly chosen mass flow rate. In essence, the feed gate adaptation algorithm computes the optimal gate angle settings needed for different application rates in real time, as if the calibration tool is incorporated in the spreader itself. In practice it is possible to record the gate angle settings for the each mass flow rate and simply use these to generate electronic spreading tables without further sensor use. In fact, to start the spreader initial gate angle settings are needed to avoid a fertilizer spill while the machine is stationary and this setting will in practice be obtained from a table created a priori.

However practical the usage of spreading tables may be, it is advised to integrate the sensor and feed gate adaptation algorithm into the spreader design since the particle properties may vary due to material changes (real time blending), and segregation effects. In addition, the implemented feedback system allows for changing the swath width and the rotational disc velocities to create tailor made patterns without recalibration of the spreader. 


\section{Acknowledgements}

The authors would like to express their sincere gratitude to the researchers and students at Cemagref, Groupement de Clermont-Ferrand, in Montoldre, France for their support and participation in this project.

\section{References}

Etschberger, 2001. Controller Area Network. IXXAT Press, 88250 Weingarten, Germany.

Fulton, J.P., S.A. Shearer, G. Chabra and S.F. Higgins. 2001. Performance Assessment And Model Development Of A Variable-Rate, Spinner-Disc Fertilizer Applicator. Transactions of the ASAE, 44(5):1071-1081.

Fulton, J.P. S. A. Shearer, S. F. Higgins, D. W. Hancock, T. S. Stombaugh. 2004. Distribution Pattern Variability of Granular VRT Applicators. Transactions of the ASAE. 48(6): 20532064.

Grift, T.E., and J.W. Hofstee. 1997. Measurement of Velocity and Diameter of Individual Fertilizer Particles by an Optical Method, J. of Agricultural Engineering Research, 66(3):235-238.

Grift, T.E. 2000. Spread Pattern Analysis Tool (SPAT), Part 1. Development and Theoretical Examples. Transactions of the ASAE, 43(6):1341-1350.

Grift, T.E., and J.W. Hofstee. 2002. Testing an Online Spread Pattern Determination Sensor on a Broadcast Fertilizer Spreader. Transactions of the ASAE. 45(3):561-567

Helms, R. S., T. J. Siebenmorgen, and R. J. Norman. 1987. The Influence of Uneven Preflood Nitrogen Distribution on Grain Yields of Rice. Arkansas Farm Research, University of Arkansas, March-April.

Inns, F. M., and A. R. Reece. 1962. The theory of the centrifugal distributor. II: Motion on the disc, off-center feed. Journal of Agricultural Engineering Research 7(4):345-353.

Mennel, R. M., and A. R. Reece. 1963. The theory of the centrifugal distributor. III: Particle trajectories. Journal of Agricultural Engineering Research 7(3):78-84.

Parish, R.L., 1999a. The Effect of Spreader Fill Level on Delivery Rate. Applied Engineering in Agriculture. 15(6):647-648.

Parish, L. R. 1999b. Granular spreaders: Selection, calibration, testing and use. Louisiana Agricultural Experiment Station.

Parish, R.L., 2002a. Broadcast Spreader Pattern Sensitivity to Impeller/Spout height and PTO Speed. Applied Engineering in Agriculture. 18(3):297-299.

Parish, R.L., 2002b. Rate Setting Effects on Fertilizer Spreader Distribution Patterns. Applied Engineering in Agriculture. 18(3):301-304. 